\title{
Successful treatment of gastrointestinal stromal tumor with multiple liver metastases with radiofrequency ablation and imatinib: A case report
}

\author{
CHUNCHUAN FU, NIANZHOU LIU, QINGSONG DENG, YUNHUA TAN, KUANSHENG MA and PING BIE
}

Institute of Hepatobiliary Surgery, Southwest Hospital, Third Military Medical University, Chongqing 400038, P.R. China

Received June 8, 2014; Accepted March 2, 2015

DOI: $10.3892 / \mathrm{ol} .2015 .3285$

\begin{abstract}
Gastrointestinal stromal tumors (GISTs) are the most common mesenchymal tumor of the gastrointestinal tract, and typically metastasize to the liver. In patients with recurrent metastatic GISTs, no single treatment is effective. The current study reports a case of GIST with 27 metastases in the liver, which was successfully treated using radiofrequency ablation (RFA) combined with surgical resection and imatinib therapy. The patient remains tumor-free 36 months after the first RFA treatment. This case suggests that comprehensive therapy including surgical resection, RFA and imatinib, may be an effective strategy for the treatment of GIST with multiple liver metastases.
\end{abstract}

\section{Introduction}

Gastrointestinal stromal tumors (GISTs) are rare neoplasms with an estimated prevalence of 10-14 per 1,000,000. GISTs are mesenchymal tumors of the gastrointestinal tract, which occur most frequently in the stomach $(60 \%)$ and small intestine (30\%), and are less frequently observed in the rectum (4\%), colon and appendix (1-2\%), and duodenum (4-5\%). In rare cases, GISTs may occur outside of the gastrointestinal tract, including in the greater omentum and mesentery $(1,2)$. All GISTs are currently regarded to be potentially malignant, with varying degrees of malignancy. GISTs in the mesentery and the small intestine are more aggressive than those at other sites, and have a higher degree of malignancy and poorer prognosis than GISTs of the stomach (3). A comprehensive treatment strategy, including surgery, radiofrequency ablation (RFA) and imatinib mesylate therapy, is effective in treating liver metastases (4). RFA has also been reported to be an effective treatment modality for

Correspondence to: Professor Kuansheng Ma, Institute of Hepatobiliary Surgery, Southwest Hospital, Third Military Medical University, 30 Gaotanyan Main Street, Chongqing 400038, P.R. China

E-mail: makuansheng@vip.sina.com

Key words: radiofrequency ablation, gastrointestinal stromal tumor, liver metastasis imatinib-resistant GIST with liver metastases (5). The present study describes a case of mesenteric GIST with 27 liver metastases, which was successfully treated by RFA combined with surgical resection and imatinib; this is the greatest number of tumors treated with the most RFA sessions for GISTs reported to date. Written informed consent was obtained from the patient.

\section{Case report}

A 47-year-old female was admitted to Southwest Hospital, Third Military Medical University (Chongqing, China) on April 13, 2011, 1 year following surgical treatment for stromal tumors of the small intestinal mesentery. Prior to this admission, resection of small bowel mesenteric stromal tumors, and subtotal resection of the jejunum and intestinal anastomosis had been performed at the Department of Gastrointestinal Surgery, The Affiliated Hospital of Zunyi Medical College (Zunyi, China) on 27th April, 2010. The surgery had revealed a tumor of $\sim 18 \times 15 \mathrm{~cm}^{2}$ in size, located inside the mesentery in the upper jejunal segment. Postoperative pathological examination confirmed a diagnosis of stromal tumors of the small bowel mesentery. For financial reasons, the patient had declined imatinib mesylate or other molecular-targeted therapies following surgery. After 1 year, color Doppler ultrasonography and enhanced computed-tomography (CT) imaging revealed multiple solid, space-occupying lesions in the liver as a result of routine follow-up (Fig. 1). These findings, in combination with the patient's history of GIST, indicated a diagnosis of liver metastases.

In May 2011, subtotal resection of the left liver and intraoperative ultrasound-guided RFA of four metastases in the right liver were conducted with the patient under general anesthesia. The postoperative pathological examination revealed predominantly fusiform tumor cells (Fig. 2) and six mitoses per 50 high power fields, confirming a diagnosis of malignant stromal tumors in the liver. Immunohistochemical evaluation showed that the tumor cells were positive for CD117, CD34, smooth muscle actin and Discovered on GIST-1 (Fig. 3). The patient was discharged on postoperative day 13. On June 20, 2011, a follow-up examination was conducted using color Doppler ultrasonography and enhanced CT imaging. Ultrasonography revealed 10 liver metastases in right liver, with a tumor size ranging from 7-22 $\mathrm{mm}$, and the patient was readmitted for RFA treatment. All tumors were ablated 


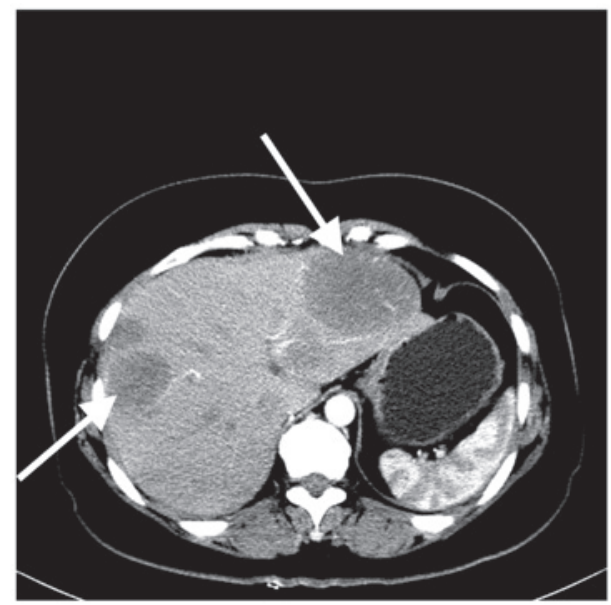

Figure 1. Abdominal enhanced computed tomography revealed multiple space-occupying lesions in the liver (arrows).

percutaneously under local or general anesthesia. Ultrasound or contrast-enhanced ultrasound was used for tumor visualization. No liver metastases were observed on postoperative CT imaging. On September 5, 2011, a follow-up examination revealed seven liver metastases, the largest measuring $7.8 \mathrm{~cm}$ in diameter (Fig. 4A). The patient was readmitted for further RFA, and postoperative ultrasonography revealed the lesion to be hyperechoic (Fig. 4B). The patient subsequently commenced therapy with oral imatinib (400 mg/day; Gleevec, Novartis, Switzerland); this regimen has been continued to the present day.

During follow-up examinations in January and May 2012, color Doppler ultrasonography revealed five liver metastases, and CT imaging revealed one liver metastasis. The patient subsequently underwent further RFA. In total, two surgeries were required to treat all tumors; three tumors were ablated for the first time, and the remaining nodules were completely destroyed for the second time. An abdominal CT scan (Fig. 4C) conducted on May 30, 2013, and contrast-enhanced ultrasonography on February 25, 2014, (Fig. 4D) revealed that the metastatic liver lesions had disappeared following RFA treatment and no new lesions were observed. At present, the patient has undergone 36 months of follow-up since the initial treatment with RFA, and has been hospitalized five times for the further RFA treatment of a total of 27 metastases, which ranged in diameter from $7-78 \mathrm{~mm}$. The patient is currently stable and in good health. The most recent contrast-enhanced ultrasonography of the liver and abdominal CT imaging indicate no new or recurrent lesions.

\section{Discussion}

Liver metastasis is one major cause of mortality among GIST patients. Following total resection of the original tumor, liver metastases typically occur in $55 \%$ of patients (6). The majority of GIST liver metastases have complete capsules, with clear boundaries between tumor and healthy liver tissues. Surgical resection is the primary treatment for liver metastasis, and resection of the GIST itself may be curative. Surgical resection has a 5-year survival rate of $34 \%$, and a median survival time of 36 months (7). However, the rate of tumor recurrence

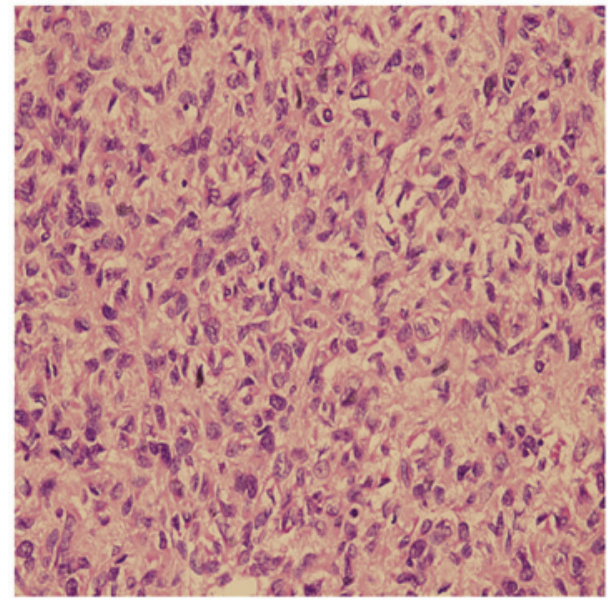

Figure 2. Liver metastases primarily composed of fusiform cells (hematoxylin and eosin staining). Magnification, $\mathrm{x} 400$.

following liver resection may exceed $70 \%(7,8)$. As only a small minority of patients are able to undergo repeated liver resection, satisfactory outcomes are difficult to achieve with surgical treatment alone.

RFA has been widely used for the treatment of liver tumors; it has the advantage of easy administration that can be repeated with minimal trauma and a low rate of complication (9). However, few reports of RFA treatment for GIST liver metastases have been published. Yamanaka et al (10) reported the successful treatment of a total of 21 liver metastases (maximum diameter, $2.2 \pm 1.1 \mathrm{~cm}$; range, 1.2-4.2 cm) from seven cases of GIST using CT-guided RFA, with a GIST-related 5 -year survival rate of $100 \%$. Jones et al (11) reported 13 cases of RFA treatment of GIST liver metastases, and a post-RFA 2-year survival rate of $77 \%$. This indicates that repeated RFA may slow the rate of liver metastasis. In the present case, a small number of asymptomatic pleural effusions in the right chest were observed following two RFA sessions, and disappeared without treatment, suggesting that RFA is an effective and safe way to treat liver metastases from GIST.

Since 2000, the development and use of the tyrosine kinase inhibitor, imatinib mesylate has greatly improved molecular-targeted therapy for GIST. The c-kit expressed by the majority of GISTs is the protein product of the c-kit proto-oncogene; imatinib inhibits the enzymatic activity of c-kit, suppresses cell proliferation and restores apoptosis. It has been used successfully to treat unresectable and metastatic GIST, and oral administration of imatinib produces satisfactory outcomes in the majority of c-kit-positive patients $(12,13)$. The adverse effects of imatinib are mild and generally tolerable, and include skin rash, edema, periorbital edema, diarrhea, nausea, vomiting and bleeding sclera. Few patients experience complications severe enough to merit the discontinuation of treatment. The National Comprehensive Cancer Network recommends imatinib as a first-line treatment for unresectable and metastatic GIST, and patients with a medium to high risk of metastasis are advised to receive at least 1 year of imatinib treatment following surgery (14).

A number of studies indicate good efficacy of combined RFA and imatinib therapy for GIST liver metastases. Pawlik et al (15) reported 36 cases of GIST liver metastases, 

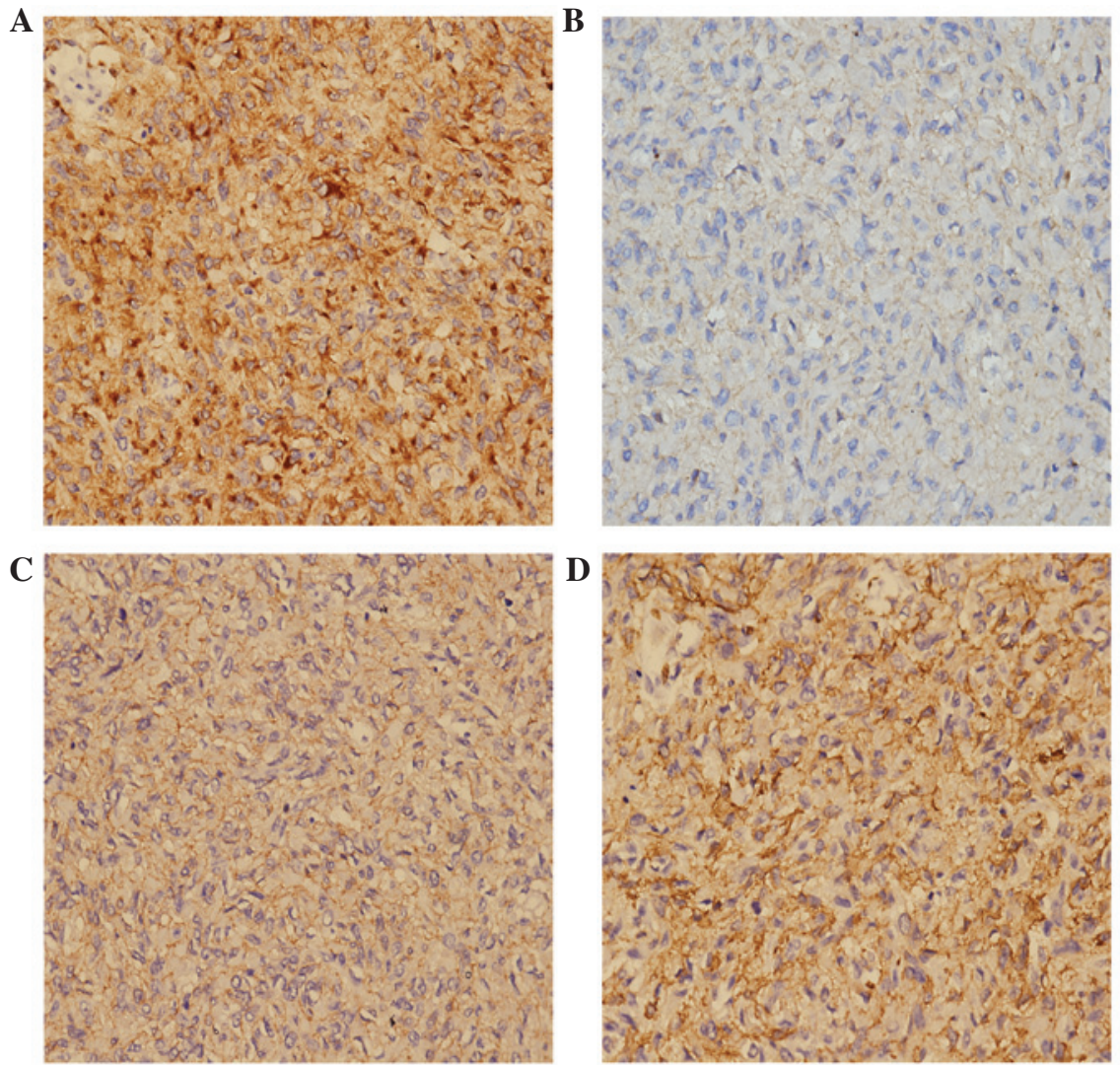

Figure 3. Immunohistochemical staining of gastrointestinal stromal tumor liver metastases. (A) CD117; (B) CD34; (C) Discovered on GIST-1; (D) Smooth muscle actin. Magnification, $\mathrm{x} 400$.

A

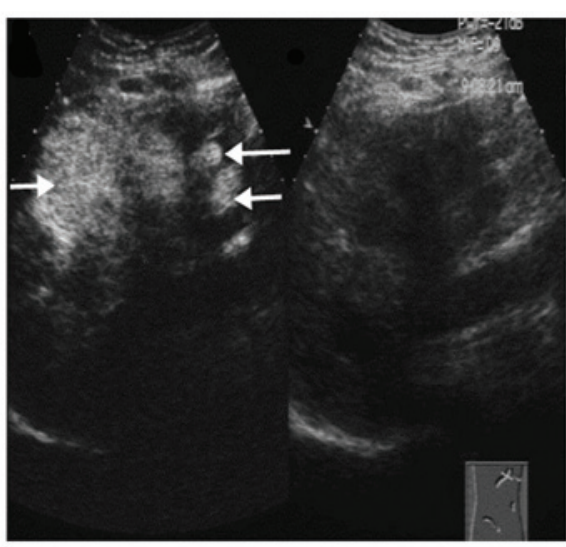

$\mathbf{C}$

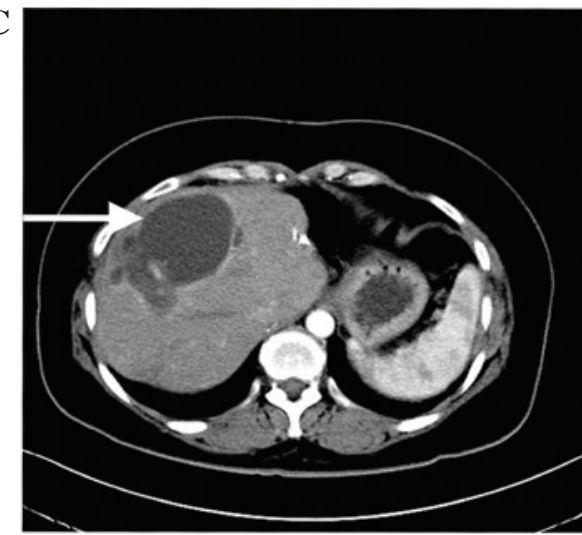

B

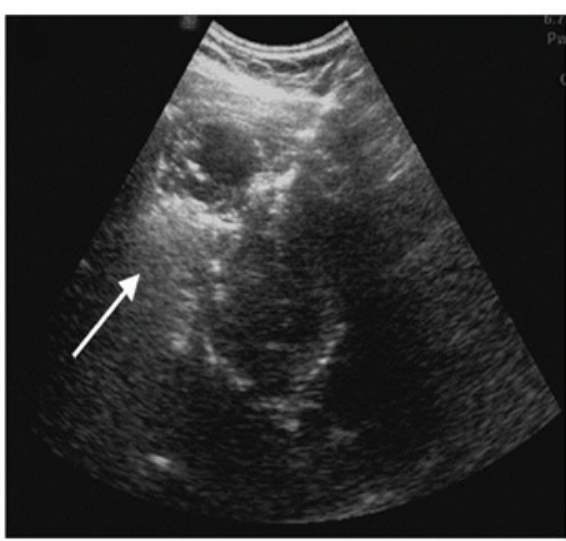

D

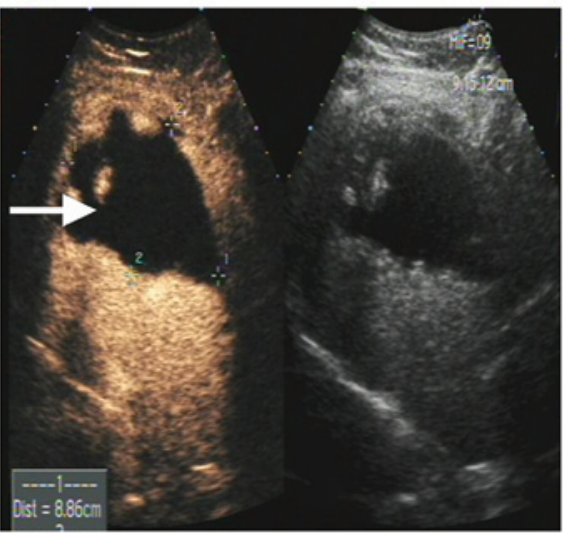

Figure 4. (A) Preoperative contrast-enhanced ultrasonography revealed multiple hyperechoic tumors in the right liver (largest, 78x53 mm). (B) Ultrasonography immediately following radiofrequency ablation showed the tumor as a hyperechoic region. (C) Abdominal enhanced computed tomography revealed that the initial liver tumors had been replaced by non-enhanced regions (i.e. ablation zones). (D) Contrast-enhanced ultrasonography revealed multiple contrast agent filling defects in the right liver. No liver metastases were observed. 
in which the majority of patients who had received RFA combined with imatinib therapy had a significantly longer median survival compared with the patients who only received RFA. A further case of combined RFA and imatinib treatment for liver metastases was reported by Ishikawa et al (4); in this study, the patient experienced $>28$ months of progression-free survival. The use of imatinib in the absence of RFA has limited efficacy with regard to the elimination of tumors and sustained tumor-free survival. Although the tumor recurrence rate following RFA is high, it is valuable in reducing the tumor load, delaying local tumor growth and controlling development of the disease. This allows the opportunity for patients to maintain tumor-free survival with oral imatinib therapy.

In the present case, RFA combined with imatinib was used to treat the largest number of liver metastases observed in a single GIST patient to date. At 2 years following the most recent RFA treatment, the patient continues to take imatinib orally, exhibiting long-term tumor-free survival with satisfactory therapeutic efficacy.

In conclusion, RFA combined with imatinib may be an effective therapy in cases of multiple liver metastases arising from GISTs. However, a large-sample study with appropriate inclusion criteria is required to verify and further optimize this treatment strategy. This study was approved by the Research Ethics Committee of the Southwest Hospital, which is affiliated with The Third Military Medical University. Written informed consent was obtained from the patient.

\section{Acknowledgements}

The authors would like to thank the Southwest Hospital Department of Information.

\section{References}

1. Miettinen M and Lasota J: Gastrointestinal stromal tumors: pathology and prognosis at different sites. Semin Diagn Pathol 23: 70-83, 2006.

2. Miettinen M and Lasota J: Gastrointestinal stromal tumors: review on morphology, molecular pathology, prognosis, and differential diagnosis. Arch Pathol Lab Med 130: 1466-1478, 2006.
3. Reith JD, Goldblum JR, Lyles RH and Weiss SW: Extragastrointestinal (soft tissue) stromal tumors: an analysis of 48 cases with emphasis on histologic predictors of outcome. Mod Pathol 13: 577-585, 2000.

4. Ishikawa A, Teratani T, Ono S, et al: A case of gastrointestinal stromal tumor with liver and bone metastases effectively treated with radiofrequency ablation and imatinib mesylate. Nihon Shokakibyo Gakkai Zasshi 103: 1274-1279, 2006 (In Japanese).

5. Minami T, Sato S, Watanabe Y, et al: Successful treatment of a gastrointestinal stromal tumor with liver metastases in a case that tolerated imatinib administration, by radiofrequency ablation using contrast-enhanced ultrasonography. Nihon Shokakibyo Gakkai Zasshi 107: 442-448, 2010 (In Japanese)

6. DeMatteo RP, Lewis JJ, Leung D, Mudan SS, Woodruff JM and Brennan MF: Two hundred gastrointestinal stromal tumors: recurrence patterns and prognostic factors for survival. Ann Surg 231: 51-58, 2000.

7. Nunobe S, Sano T, Shimada K, Sakamoto Y and Kosuge T: Surgery including liver resection for metastatic gastrointestinal stromal tumors or gastrointestinal leiomyosarcomas. Jpn J Clin Oncol 35: 338-341, 2005.

8. Gomez D, Al-Mukthar A, Menon KV, Toogood GJ, Lodge JP and Prasad KR: Aggressive surgical resection for the management of hepatic metastases from gastrointestinal stromal tumours: a single centre experience. HPB (Oxford) 9: 64-70, 2007.

9. Li L, Zhang J, Liu X, Li X, Jiao B and Kang T: Clinical outcomes of radiofrequency ablation and surgical resection for small hepatocellular carcinoma: a meta-analysis. J Gastroenterol Hepatol 1: $51-58,2012$.

10. Yamanaka T, Takaki H, Nakatsuka A, et al: Radiofrequency ablation for liver metastasis from gastrointestinal stromal tumor. J Vasc Interv Radiol 24: 341-346, 2013.

11. Jones RL, McCall J, Adam A, et al: Radiofrequency ablation is a feasible therapeutic option in the multi modality management of sarcoma. Eur J Surg Oncol 36: 477-482, 2010.

12. Gastrointestinal Stromal Tumor Meta-Analysis Group (MetaGIST): Comparison of two doses of imatinib for the treatment of unresectable or metastatic gastrointestinal stromal tumors: a meta-analysis of 1,640 patients. J Clin Oncol 28: $1247-1253,2010$

13. Blanke CD, Demetri GD, von Mehren M, et al: Long-term results from a randomized phase II trial of standard- versus higher-dose imatinib mesylate for patients with unresectable or metastatic gastrointestinal stromal tumors expressing KIT. J Clin Oncol 26: 620-625, 2008

14. Demetri GD, von Mehren M, Antonescu CR, et al: NCCN Task Force report: update on the management of patients with gastrointestinal stromal tumors. J Natl Compr Canc Netw 8: S1-S41, 2010.

15. Pawlik TM, Vauthey JN, Abdalla EK, Pollock RE, Ellis LM and Curley SA: Results of a single-center experience with resection and ablation for sarcoma metastatic to the liver. Arch Surg 141: 537-543, 2006. 